\title{
miR-146a promotes growth of osteosarcoma cells by targeting ZNRF3/GSK-3 $\beta / \beta$-catenin signaling pathway
}

\author{
Chun Zhou ${ }^{1}$, Chang-Qing Jiang ${ }^{1}$, Zhen Zong ${ }^{1}$, Jia-Chen Lin ${ }^{1}$ and Li-Feng Lao ${ }^{1}$ \\ ${ }^{1}$ Department of Orthopaedic Surgery, Renji Hospital, School of Medicine, Shanghai Jiao Tong University, Shanghai, China \\ Correspondence to: Li-Feng Lao, email: laolifeng03@163.com \\ Keywords: miR-146a-5p, growth, ZNRF3, osteosarcoma, recurrence \\ Received: April 09, $2017 \quad$ Accepted: June 19, $2017 \quad$ Published: July 19, 2017 \\ Copyright: Zhou et al. This is an open-access article distributed under the terms of the Creative Commons Attribution License 3.0 \\ (CC BY 3.0), which permits unrestricted use, distribution, and reproduction in any medium, provided the original author and source \\ are credited.
}

\section{ABSTRACT}

\begin{abstract}
MicroRNA-146a-5p (miR-146a) functions as a tumor suppressor or oncogene involved in multiple biological processes. But, the underlying molecular mechanisms by which miR-146a contributes to osteosarcoma (OS) remain unclear. The correlation of miR-146a expression with clinicopathologic characteristics and prognosis of OS patients was analyzed by Kaplan-Meier and Cox regression analysis. Cell growth in vitro and in vivo was assessed by MTT, cell colony formation and animal models. The target of miR-146a was identified by bioinformatics software and gene luciferase reporter. As a result, miR-146a expression was substantially elevated in OS tissues and was positively associated with the tumor size $(P=0.001)$ and recurrence $(P=0.027)$ of OS patients. Moreover, knockdown of miR-146a suppressed cell proliferation and colony formation in vitro and in vivo. In addition, zinc and ring finger 3 (ZNRF3) was identified as a direct target of miR-146a in OS cells, and was negatively correlated with miR-146a expression in OS tissues. Overexpression of ZNRF3 inhibited cell growth and rescued the tumor-promoting role of miR-146a via inhibition of GSK-3 $\beta / \beta-$ catenin signaling pathway. Taken together, miR-146a may function as an oncogene

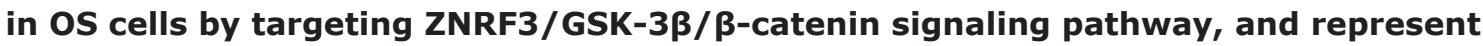
a promising biomarker for OS patients.
\end{abstract}

\section{INTRODUCTION}

Osteosarcoma (OS) is considered to be an aggressive bone neoplasm and occurs mainly in children and young adults [1]. Although the great progress has been made in surgical treatment combined with multidrug chemotherapy, the five-year survival rate and recurrence risk of OS patients have not been significantly improved due to the tumor local relapse and metastases [2]. Many molecular alterations in critical signal transduction pathways contribute to the OS pathogenesis via promoting the proliferation and metastasis [3]. Herein, identifying the key molecular targets involved in OS progression will be highlighted for diagnosis and therapy of OS patients.

MicroRNAs (miRNAs) as noncoding RNAs have participated in multiple biological and pathogenesis of diseases by regulating gene expression. Dysregulation of miRNAs as oncogenes or tumor suppressors is involved in multiple steps of the oncogenic process [4]. On the one hand, miR-146a expression is found upregulated in gastric cancer [5], breast cancer [6], premalignant thymocytes [7] and thyroid cancer [8], and the rs2910164 polymorphism in miR-146a is related with the incidence of melanoma [9]. Ectopic expression of $\mathrm{miR}-146 \mathrm{a}$ results in cell proliferation $[8,10]$, migration [10], invasion and metastasis [11] in some cancers. Moreover, some key pathways mediate miR-146a promoting tumor progression. miR-146a facilitates growth and development of melanoma by activation of Notch signaling [12], reduces cell apoptosis in acute promyelocytic leukemia by Smad4 signaling [13] and enhances tumorigenic potential of breast cancer [14]. These data indicate that miR-146 may function as an oncogene involved in tumor progression. 
On the other hand, miR-146a expression is decreased in various cancers, such as NK/T cell lymphoma [15], gastric cancer [16], castration-resistant prostate cancer [17], head and neck squamous cell carcinoma [18], hepatocellular carcinoma [19], penile squamous cell carcinoma [20] and non-small cell lung cancer [21]. Enforced expression of miR-146a suppresses cell proliferation and invasion [15-19, 21-24) and angiogenesis via targeting EGFR [17], RAF6 [19], CCND1/2 [21], Notch1 [22] or Rac1 [24], induces cell apoptosis [15, 16, 23] and enhanced chemo-sensitivity [15]. Therefore, miR146 may function as a tumor suppressor for cancer therapy.

Despite the bi-directional role of miR-146a in cancers, its function and underlying regulation mechanism remain unclear in OS. In the present study, we found that miR-146a expression was increased in OS tissues and was positively correlated with the tumor size and high recurrence rate of OS patients. Knockdown of miR-146a suppressed cell growth in vitro and in vivo. In addition, ZNRF3 was identified as a direct target of miR-146a in OS cells, and had a reverse correlation with miR-146a expression in OS tissues. Overexpression of ZNRF3 inhibited cell growth and attenuated the tumor-promoting effects of miR-146a via inhibition of GSK-3 $\beta / \beta$-catenin signaling, indicating that miR-146a may function as a tumor suppressor and represent a potential biomarker for OS patients.

\section{RESULT}

\section{miR-146a expression was upregulated in human OS tissues}

To confirm the expression level of miR-146a in human OS tissues, we used the 2015 The cancer Genome Atlas (TCGA) gene sequencing data (https://genomecancer.ucsc.edu/) to examine miR-146a expression in OS tissues, indicating that miR-146a was highly expressed in OS tissues $(n=237)$ compared to the adjacent normal tissues $(n=25)$ (Figure 1A). miR-146a expression was also upregulated in OS tissues $(n=25)$ compared with the pairmatched normal tissues (n-25) (Figure 1B). To uncover the link between miR-146a expression and tumor growth in OS tissues, we detected the expression of miR-146a in OS with tumor size $\geq 3 \mathrm{~cm}(\mathrm{n}=74)$ or tumor size $<3 \mathrm{~cm}(\mathrm{n}=119)$, indicating that miR-146a expression was substantially upregulated in OS with tumor size $\geq 3 \mathrm{~cm}$ compared with those with tumor size $<3 \mathrm{~cm}$ (Figure 1C).

\section{The correlation of miR-146a expression with clinicopathologic features and prognosis of OS patients}

We further analysed the association of miR-146a expression with clinicopathologic characteristics and
A

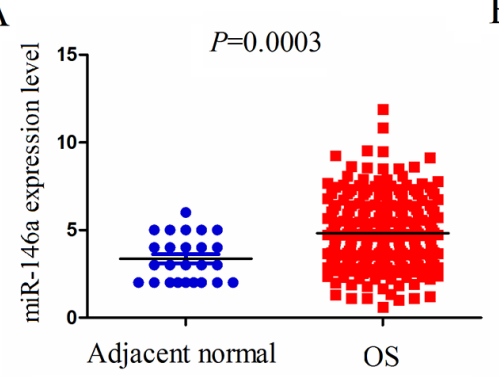

$\mathrm{D}$

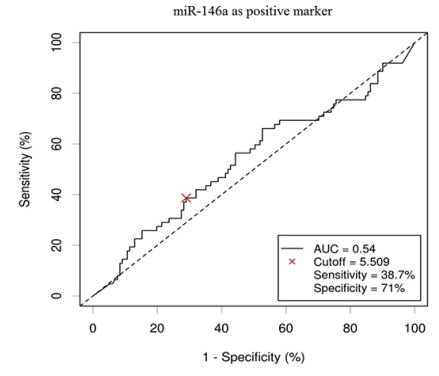

B

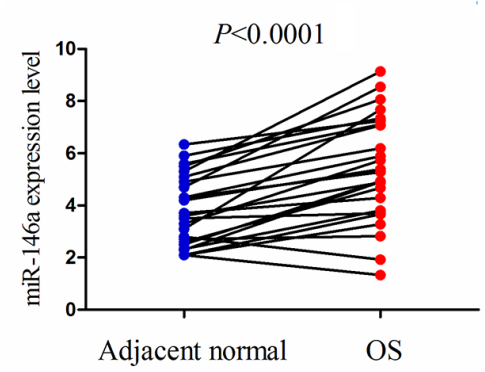

E

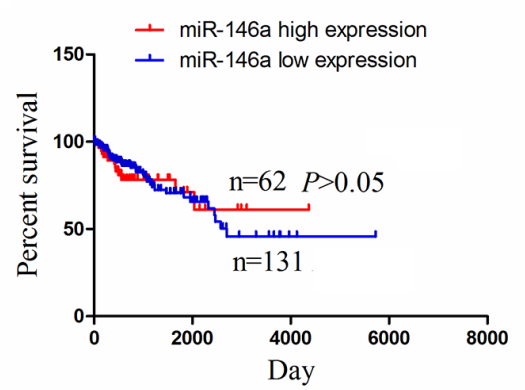

$\mathrm{C}$

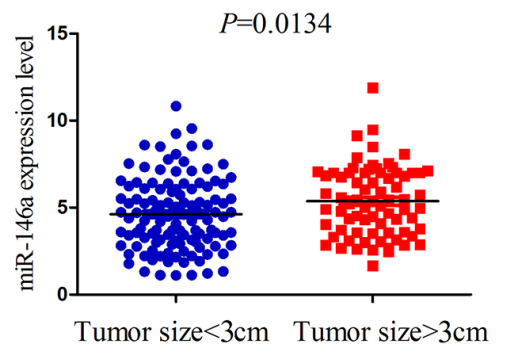

F

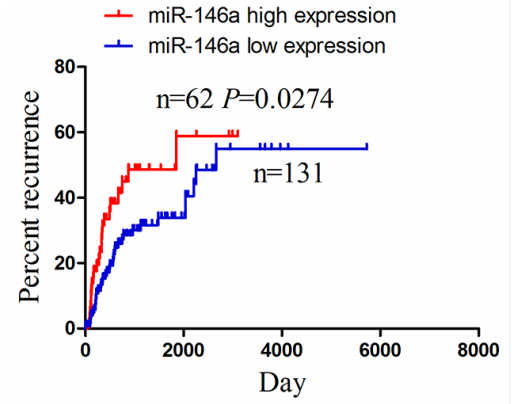

Figure 1: The expression of miR-146a was upregulated in OS tissues and associated with tumor recurrence. (A) The expression of miR-146a in 237 OS patient samples and 25 normal tissues shown by a 2015 TCGA data set. (B) TCGA data analysis of the expression of miR-146a in paired OS tissues $(n=25)$. (C) The expression level of miR-146a in OS patients with tumor size $\geq 8 \mathrm{~cm}(\mathrm{n}-74)$ and tumor size $<8 \mathrm{~cm}(\mathrm{n}=119)$. (D) The cutoff value of miR-146a was determined by the patient survival time, status and miR-146 expression level. (E) The correlation of miR-146a high expression or low expression with the overall survival rate of OS patients. (F) The correlation of miR-146a high expression or low expression with the tumor recurrence rate of OS patients. 
prognosis of OS patients. As indicated in Table 1, miR146a expression was positively associated with tumor size ( $P=0.004$ ), but had no correlation with age, gender, lymph node infiltration and distant metastasis in OS patients (each $P>0.05)$. According to the overall survival time, survival status and miR-146a expression level, we used the cutoff finder (http://molpath.charite.de/cutoff/load.jsp) to define the high expression level (cutoff $\geq 5.509$ ) or low expression level (cutoff $<5.509$ ) of miR-146a in OS tissues (Figure 1D). We then analyzed the association between miR-146a high expression and overall survival and recurrence in OS patients by using Kaplan-Meier and Multivariate analysis, indicating that miR-146a expression was not an independent prognostic factor for overall survival (Supplementary Table 1) and tumor recurrence (Supplementary Table 2) in OS patients. Overall survival curve demonstrated that OS patients with miR-146a high expression had no difference in comparison with the patients with miR-146a low expression (Figure 1E), but the recurrence curve showed that the patients with miR-146a high expression had higher tumor recurrence rate than the patients with miR-146a low expression (Figure 1F).

\section{The effects of miR-146a on OS cell growth}

Having verified the positive correlation of miR-146a expression with tumor size of OS patients (Figure 1C and Table 1), we then explored the functions of miR-146a in
OS growth. We first examined the expression levels of miR-146a in different OS cell lines (U-2 OS, Saos-2, MG63, SW-1353, HOS and 143B), and found that miR-146a was highly expressed in U-2 OS and Saos-2 cell lines but lowly expressed in MG-63 and HOS cell lines (Figure 2A). Then, miR-146a shRNA vector was transfect intro U-2 OS and Saos-2 cell lines with miR-146a high expression. After transfection for 48h, miR-146a expression levels were determined by qRT-PCR, indicating a decreased miR-146a expression in sh-miR-146a group compared to NC group (Figure 2B). Then, MTT and colony formation assays showed that knockdown of miR-146a significantly reduced cell proliferation activity (Figure 2C and 2D) and colony formation (Figure 2E) in OS cells.

Meanwhile, miR-146a overexpression vector was used to transfect into MG-63 and HOS cell lines with miR-146a low expression. After transfection for 48h, miR146 a expression levels were markedly increased in miR146a group compared with NC group (Figure 3A). MTT and colony formation assays showed that overexpression of miR-146a promoted cell growth of MG-63 and HOS cells (Figure 3B and 3C).

\section{ZNRF3 was a target gene of miR-146a in OS cells}

To confirm the molecular mechanism by which miR-146a regulates cell growth in OS cells, we
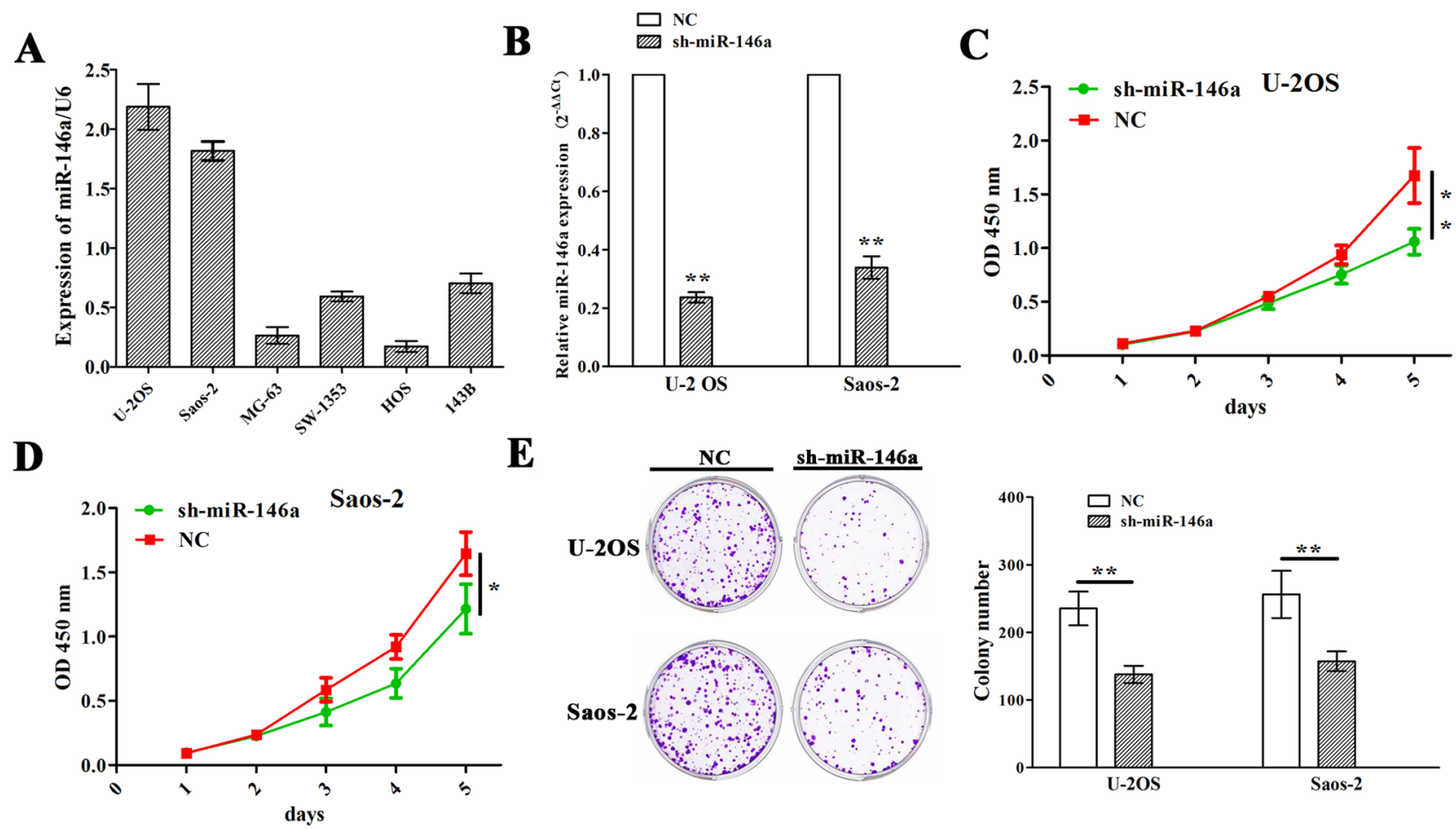

$\mathbf{E}$
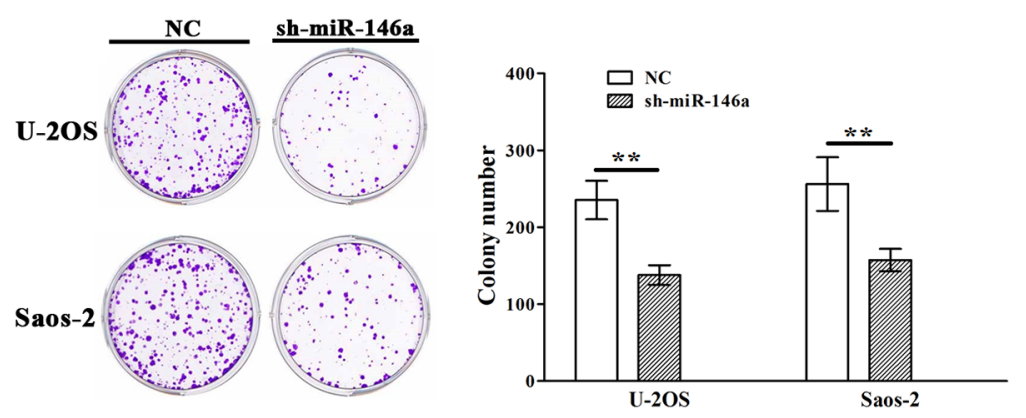

Figure 2: Knockdown of miR-146a inhibited OS cell proliferation and colony formation. (A) qRT-PCR analysis of the expression levels of miR-146a in multiple OS cell lines. (B) qRT-PCR analysis of the knockdown efficiency of sh-miR-146a in U-2OS and Saos-2 cells. (C and D) The effects of miR-146a knockdown on cell proliferation of U-2OS and Saos-2 cells indicated by MTT assay. (E) The effects of miR-146a knockdown on cell colony formation of U-2OS and Saos-2 cells. $* P<0.05, * * P<0.01$. 
Table 1: The correlation of miR-146a expression with clinicopathological factors of OS patients

\begin{tabular}{|c|c|c|c|c|}
\hline Variables & Cases (n) & miR-146a low & Expression high & $P$ value \\
\hline Total & 193 & 131 & 62 & \\
\hline \multicolumn{5}{|c|}{ Age (years) } \\
\hline$\geq 20$ & 83 & 61 & 22 & \\
\hline$<20$ & 110 & 70 & 40 & 0.148 \\
\hline \multicolumn{5}{|l|}{ Gender } \\
\hline Male & 89 & 57 & 32 & \\
\hline Female & 104 & 74 & 30 & 0.293 \\
\hline \multicolumn{5}{|c|}{ Tumor size (cm) } \\
\hline$\geq 3$ & 74 & 41 & 33 & \\
\hline$<3$ & 119 & 90 & 29 & 0.004 \\
\hline \multicolumn{5}{|c|}{ Lymph node infiltration } \\
\hline No & 97 & 69 & 28 & \\
\hline Yes & 96 & 62 & 34 & 0.331 \\
\hline \multicolumn{5}{|c|}{ Distant metastases } \\
\hline No & 168 & 115 & 53 & \\
\hline Yes & 25 & 16 & 9 & 0.657 \\
\hline
\end{tabular}

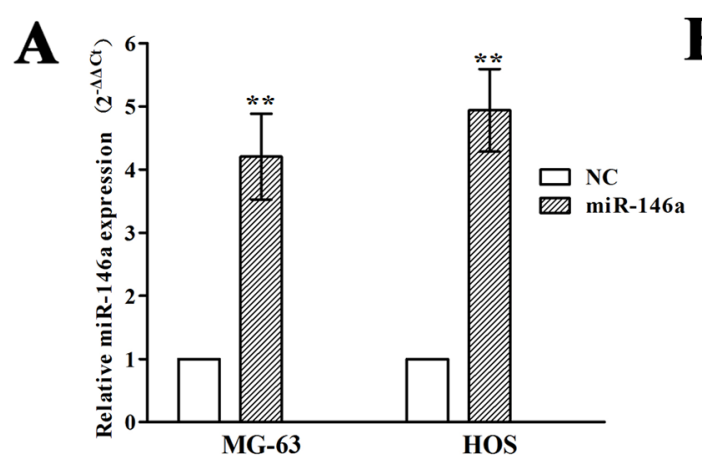

B
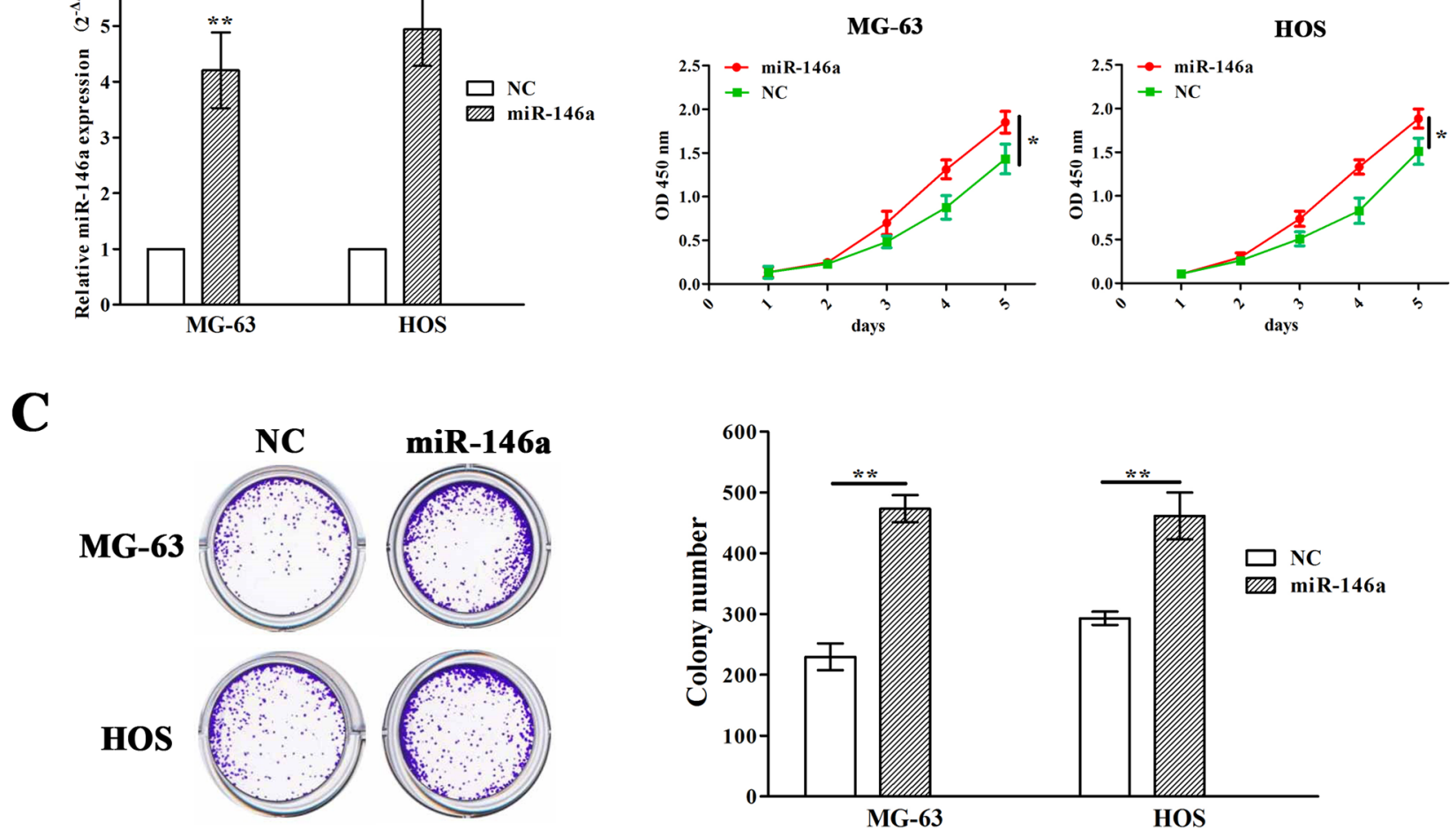

Figure 3: Ectopic expression of miR-146a promoted OS cell proliferation and colony formation. (A) qRT-PCR analysis of the transfection efficiency of lentivirus mediated miR-146a in MG-63 and HOS cells. (B) The effects of miR-146a overexpression on cell proliferation of MG-63 and HOS cells indicated by MTT assay. (C) The effects of miR-146a overexpression on cell colony formation of MG-63 and HOS cells. ${ }^{*} P<0.05, * * P<0.01$. 
identified the potential target genes of miR-146a by using prediction websites (http://starbase.sysu.edu.cn/ targetSite.phpfor). As shown in Figure 4A, under the stringent conditions including very high stringency $(\geq 5)$, cancer types $(\geq 5)$ and prediction software $(\geq 3)$, six target genes were identified to have the potential to bind with miR-146a, of which zinc and ring finger 3 (ZNRF3) was considered as the most suitable candidate target gene of the miR-146a due to its highest binding force (Supplementary Table 3). To further verify whether miR-146a directly binds to the 3' UTR of ZNRF3, the wild type 3' UTR or the mutant 3' UTR target sequences of ZNRF3 (Figure 4B) were cloned into the luciferase reporter vector in MG-63 and HOS cells. It was demonstrated that miR-146a decreased the expression levels of ZNRF3 in OS cells (Figure 4C). Moreover, miR-146a overexpression lowered the luciferase activity of wild type $3^{\prime}$ UTR of ZNRF3 in OS cells, but had no effect on that of mutation $3^{`}$ UTR of ZNRF3 (Figure 4D).

\section{ZNRF3 suppressed cell proliferation and colony formation of $\mathrm{OS}$ cells}

To demonstrate the association of miR-146a expression with ZNRF3 in OS tissues, we first measured the expression level of ZNRF3 in OS tissues $(n=237)$ and adjacent normal tissues $(n=25)$ by TCGA data, which uncovered that ZNRF3 expression was substantially downregulated in OS tissues (Figure 5A). ZNRF3 expression was also decreased in OS tissues compared with pair-matched normal tissues (Figure 5B). The correlation analysis showed the negative correlation of miR-146a with ZNRF3 expression in OS tissues (Figure 5C). Furthermore, we constructed the ZNRF3 overexpression stably transfected MG-63 and HOS cell lines, and confirmed its transfection efficiency by qRTPCR and western blotting assays (Figure 5D). Then, ectopic expression of ZNRF3 significantly repressed cell proliferation (Figure 5E) and colony formation (Figure 5F), but knockdown of ZNRF3 by sh-ZNRF3 (Figure 5G) promoted cell proliferation in OS cells (Figure 5H).

\section{ZNRF3 rescued the proliferation-promoting effects of miR-146a via GSK-3 $\beta / \beta$-catenin pathway}

To uncover the molecular mechanism by which ZNRF3 rescues the proliferation-promoting effects induced by miR-146a, we co-transfected ZNRF3 and miR-146a overexpression vectors into MG-63 and HOS cells, indicating that ZNRF3 overexpression reduced miR-146a-induced cell proliferation (Figure 6A). Moreover, the expression levels of GSK$3 \beta$ and $\beta$-catenin as potential downstream factors of ZNRF3 were detected by Western blot, indicating that miR-146a increased the activity of GSK-3 $\beta / \beta$-catenin pathway, but this effect was reversed by ZNRF3 in OS cells (Figure 6B). Then, we co-transfected sh-ZNRF3
$\mathbf{A}$

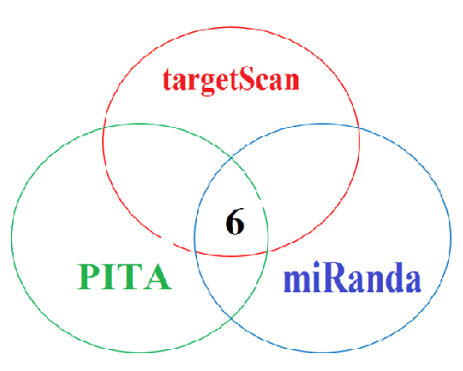

C

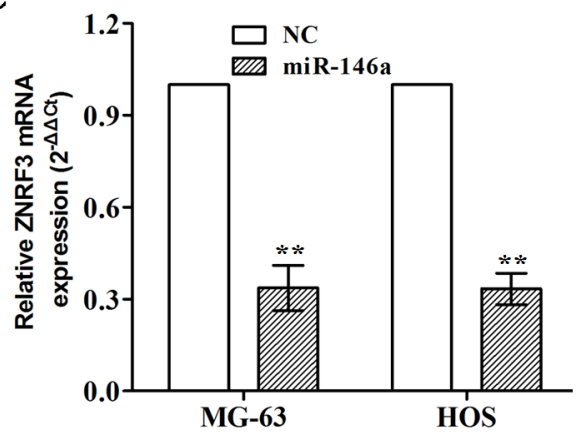

B

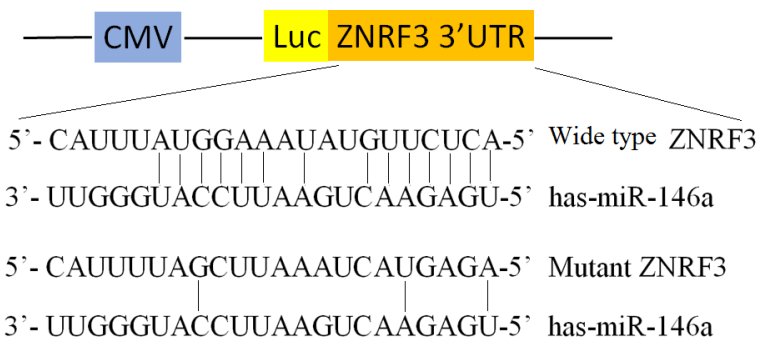

D

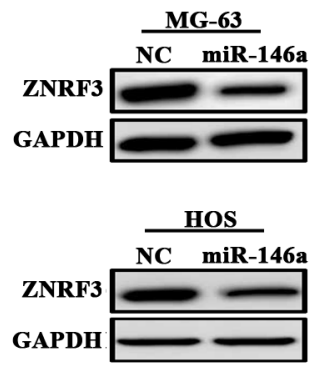

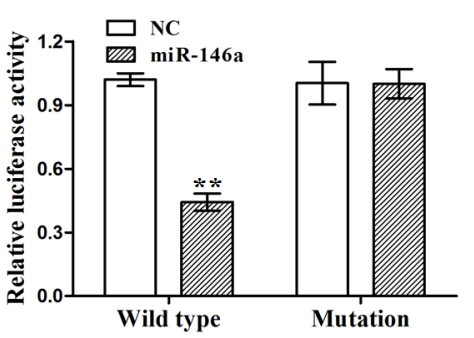

Figure 4: ZNRF3 gene was identified as a direct target of miR-146a. (A) 6 target genes of miR-146a were identified by miRNA forecasting software. (B) Diagrams demonstrated the miR-146a putative binding sites and corresponding mutant sites of ZNRF3. (C) The expression levels of ZNRF3 were examined after transfection with miR-146a by qRT-PCR and Western blotting assays in MG-63 and HOS cells. (D) Luciferase activity of ZNRF3 (wild type or mutation) was evaluated after miR-146a transfection into MG-63 and HOS cells. **P<0.01. 
and miR-146a knockdown vectors into OS U2-OS and Saos-2 cells, and found that knockdown of ZNRF3 attenuated the effects of sh-miR-146a induced increased expression of ZNRF3 (Figure 6C and 6D) and decreased proliferation of OS cells (Figure 6E).
miR-146a knockdown inhibited xenograft tumor growth

Having confirmed the proliferation-promoting role of miR-146a in OS cells in vitro, we further
$\mathbf{A}$

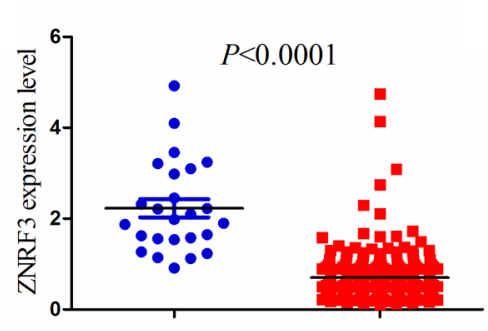

Adjacent normal
B

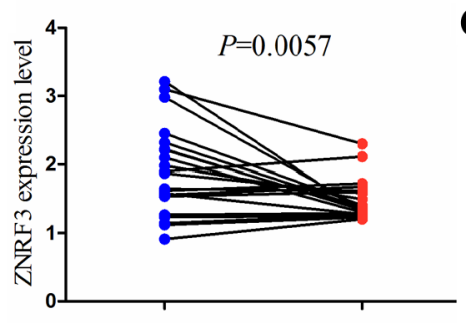

Adjacent normal OS

$\mathbf{E}$

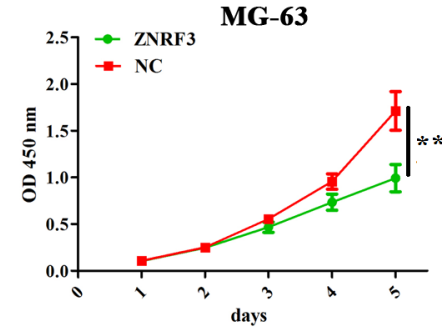

$\mathbf{F}$

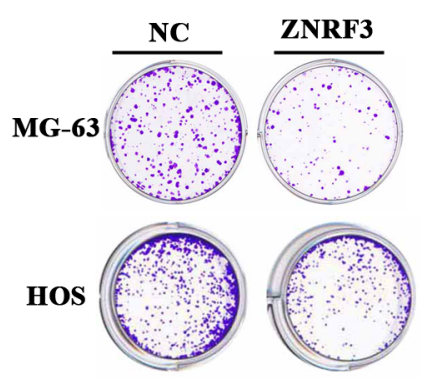

$\mathbf{H}$

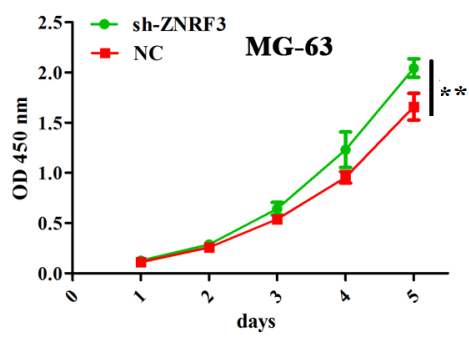

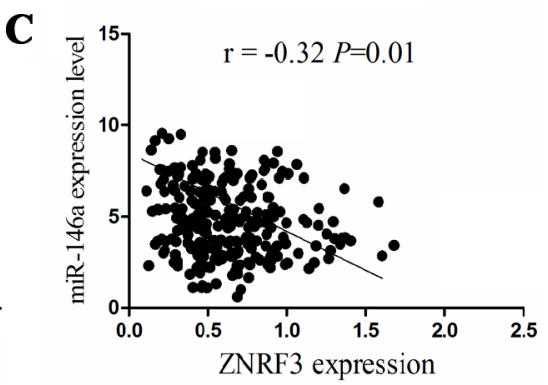
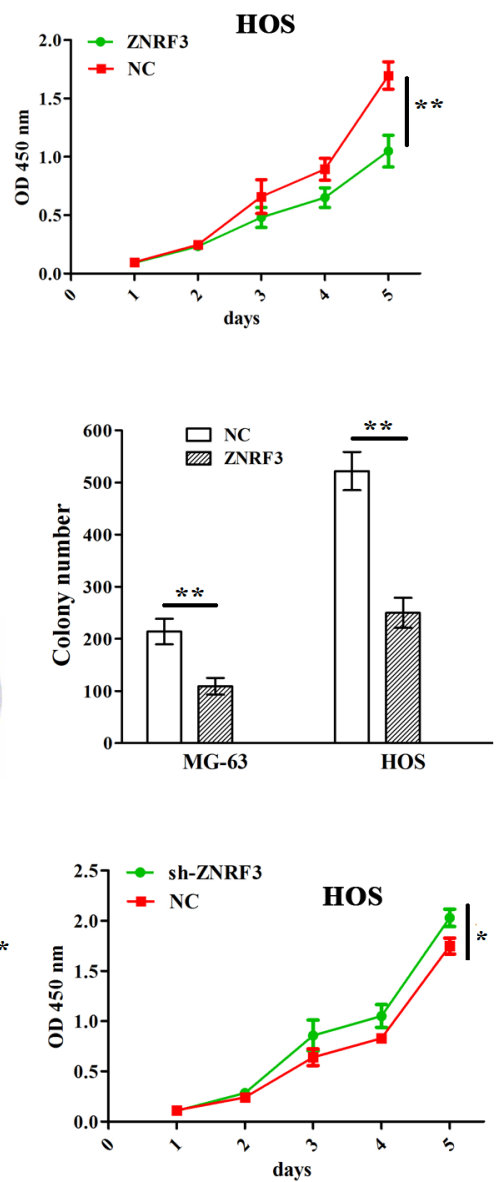

Figure 5: Enforced expression of ZNRF3 inhibited OS cell proliferation and colony formation. (A) The expression of ZNRF3 in OS tissues $(\mathrm{n}=237)$ and the adjacent normal tissues $(\mathrm{n}=25)$ shown by TCGA data. (B) The expression of ZNRF3 in paired OS tissues ( $\mathrm{n}=25)$. (C) Thecorrelation analysis showed the association of miR-146a expression with ZNRF3 in OS patients. (D) The transfection efficiency of ZNRF3 overexpression was determined by qPCR and western blot assays in MG-63 and HOS cells. (E) The effects of ZNRF3 overexpression on cell proliferation of MG-63 and HOS cells by MTT assay. (F) The effects of ZNRF3 overexpression on cell colony formation of MG-63 and HOS cells. (G) Western blot analysis of the transfection efficiency of ZNRF3 knockdown in MG-63 and HOS cells transfected with sh-ZNRF3 and NC. (H) The effects of ZNRF3 knockdown on cell proliferation of MG-63 and HOS cells by MTT assay. $* P<0.05, * * P<0.01$. 
verified the effects of miR-146a on OS cell growth in vivo. A subcutaneous U-2OS tumor model was esbablished to assess the growth activity of xenograft tumors affected by miR-146a. During the 3 weeks' investigation, the tumor length and width of the xenograft tumors was examined every other day. We found that the proliferation rates of tumors were significantly decreased by sh-miR-146a (Figure 7A and 7B). The average weight and volumes in sh-miR-146a group were lowered compared with those in $\mathrm{NC}$ group (Figure 7C and 7D). Further, western blotting analysis showed that knockdown of miR-146a downregulated the expression of ZNRF3 in xenograft tumor tissues (Figure 7E).
A

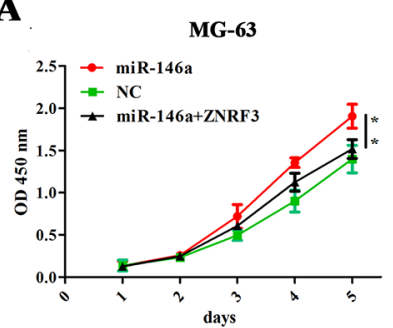

C

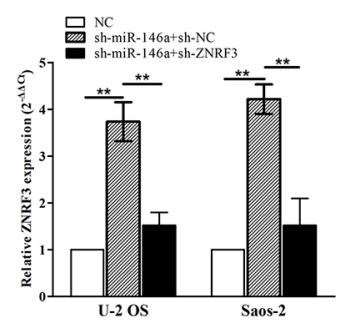

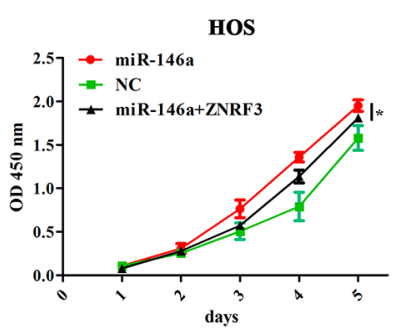

D

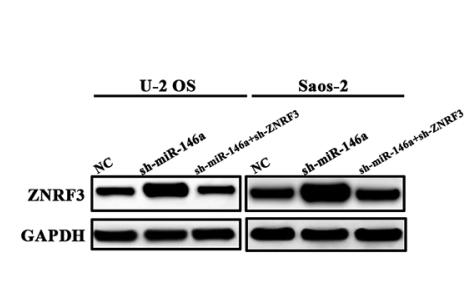

B

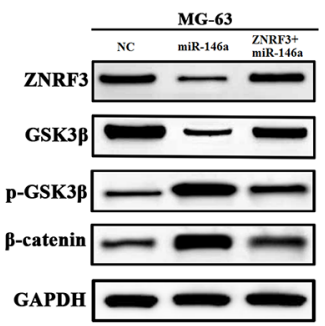

$\mathbf{E}$

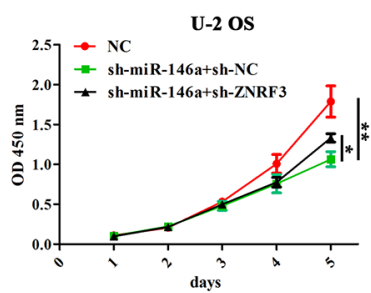

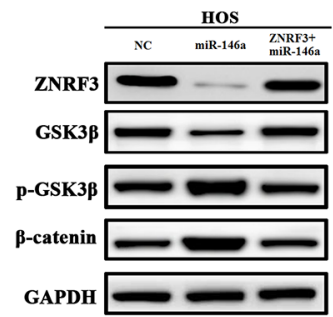

GAPDH

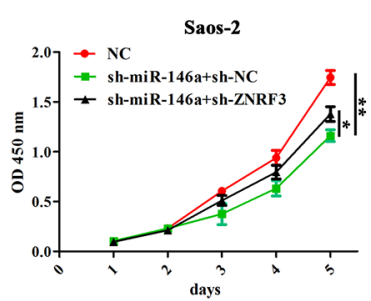

Figure 6: ZNRF3 overexpression rescued tumor proliferation-promoting effects by miR-146a. (A) The effects of ZNRF3 overexpression on cell proliferation in miR-146a-transfected MG-63 and HOS cells indicated by MTT assay. (B) The effects of ZNRF3 overexpression on the protein expression of GSK-3 $\beta / \beta$-catenin pathway in miR-146a-transfected OS cells indicated by western blotting. (C, D) Real-time PCR and western blot analysis of the expression levels of ZNRF3 in sh-ZNRF3 and sh-miR-146a co-transfected U-2 OS and Saos-2 cells. (E) MTT analysis of cell proliferative activities of U-2 OS and Saos-2 cells co-transfected by sh-ZNRF3 and sh-miR-146a. $* P<0.05, * * P<0.01$.

A

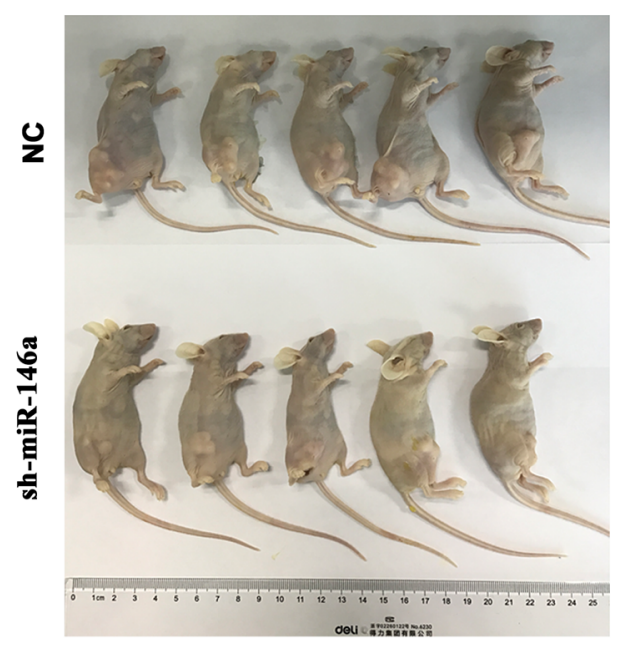

B

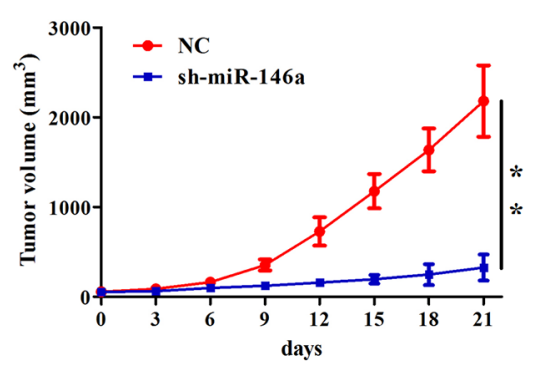

C

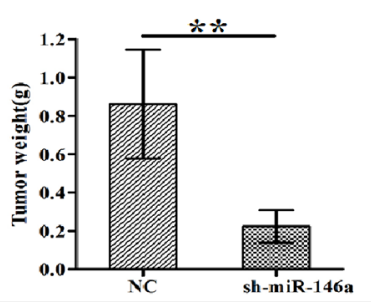

D

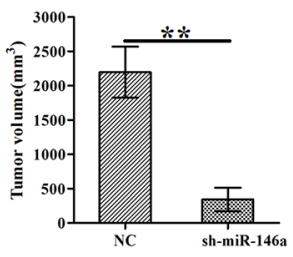

$\mathbf{E}$

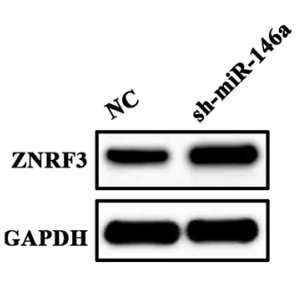

Figure 7: Knockdown of miR-146a inhibited U-2OS xenograft tumor growth. (A) Schematic representation of the subcutaneous xenograft tumor size between sh-miR-146a and NC groups. (B) The effects of miR-146a knockdown on tumor proliferation activity of U-2OS xenograft tumors. (C, D) The effects of miR-146a knockdown on the average weight and volumes in xenograft tumors on the 21 st day. (E) Western blotting analysis of the effect of miR-146a knockdown on ZNRF3 expression. ${ }^{*} * \mathrm{P}<0.01$. 


\section{DISCUSSION}

Accumulating evidence shows that miR-146a is implicated in cell growth and metastasis in various cancers, of which the dysregulation of miR-146a is associated with pathological staging, poor differentiation and distant metastasis, acting as an independent prognostic element for overall survival in gastric cancer $[25,26]$ and lung cancer [27]. In addition, miR-146a polymorphism rs2910164 is linked to the risk of esophageal cancer and colorectal cancers $[28,29]$. In this study, we analyzed the correlation of miR-146a with clinicopathologic characteristics and prognosis of OS patients, which indicated that increased expression of miR-146a was positively linked to tumor size and OS recurrence, but Kaplan-Meier analysis indicated that miR-146a was not an independent prognostic factor for overall survival and recurrence for OS patients, unveiling that miR-146a might be a potential biomarker for OS patients.

Functionally, in terms of the diversity of tissue expressions and the difference in target gene function, imiR-146a can act as an oncogene [12-14] or a tumor suppressor [21-24] in a variety of malignancies. miR-146a inhibits cell invasion in pancreatic cancer via inhibition of EGFR and NF-kB pathways [30], weakens the metastatic potential in breast cancer [31] and reduces epithelialmesenchymal transition of esophageal carcinoma [32]. But, the function of miR-146a in human OS tissues is elusive. To confirm its role in human OS, our results showed that knockdown of miR-146a inhibited growth and colony formation in vitro and in vivo, revealing the tumor-promoting role of miR-146a in OS cells.

ZNRF3 as a member of E3 ubiquitin ligases family is downregulated in gastric cancer [33] and papillary thyroid carcinoma [34] and colorectal cancer [35], and inhibits cell growth and invasion and induces apoptosis via regulation of the Wnt/ $\beta$-catenin pathway $[33,36]$. MiR-93 promotes growth of lung cancer cells via ZNRF3/Wnt/ $\beta$ catenin axis [37], and ZNRF3 suppresses the tumorigenesis and metastasis of nasopharyngeal carcinoma cells by inhibition of $\mathrm{Wnt} / \beta$-catenin signaling [38]. Intriguingly, we also found that ZNRF3 was a direct target of miR$146 \mathrm{a}$ in OS cells and was negatively correlated with miR146a expression in OS tissues; Overexpression of ZNRF3 repressed cell growth and rescued the tumor-promoting

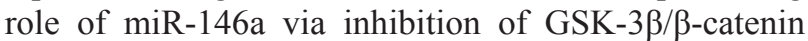

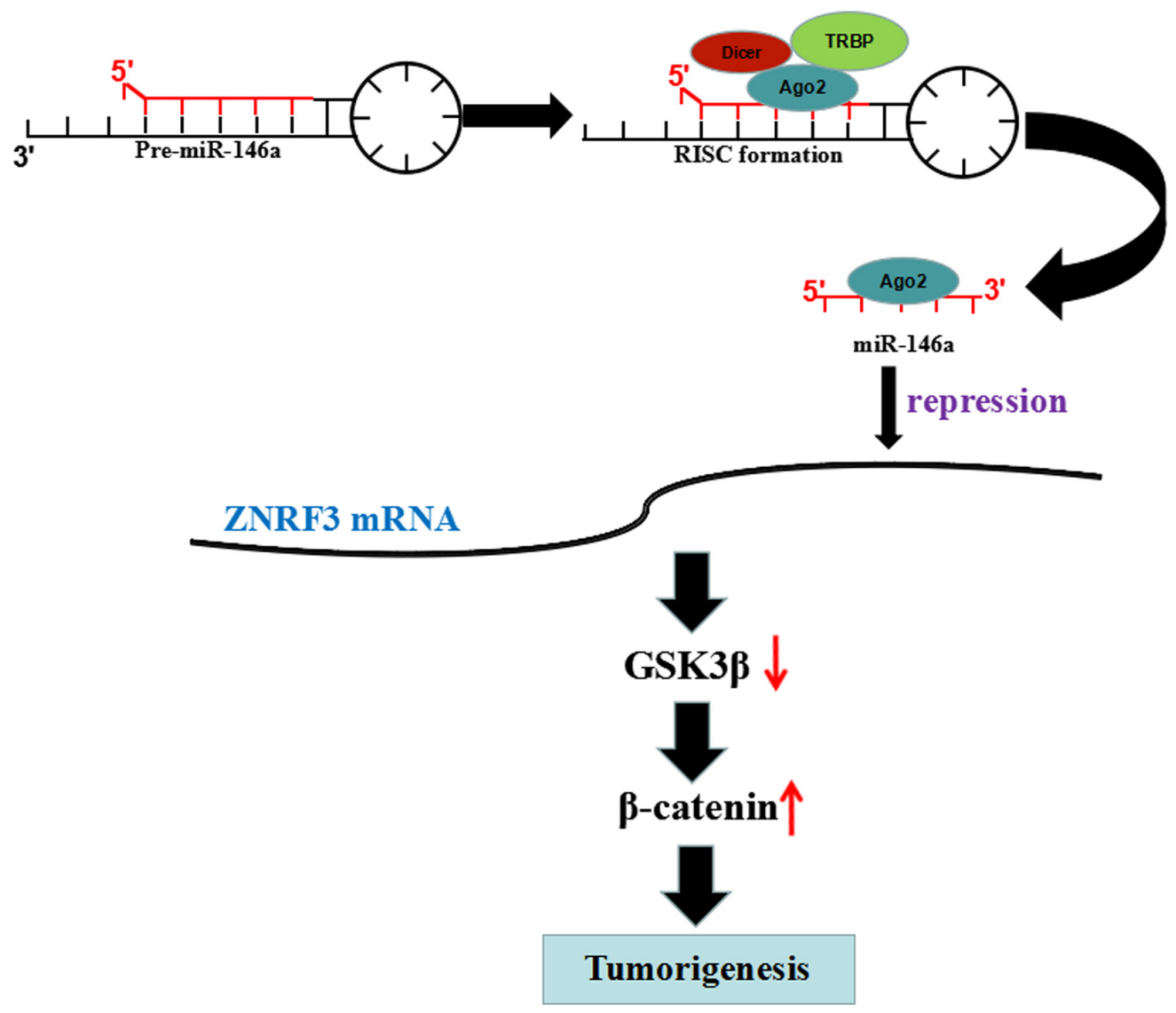

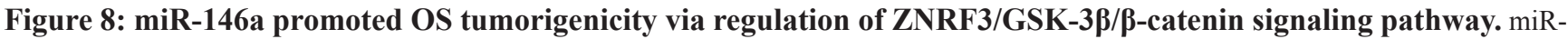
146 reduced the transcriptional level of ZNFR3 gene by binding to its 3' UTR, upregulated the expression of p-GSK-3 $\beta$ and $\beta$-catenin, but downregulated GSK-3 $\beta$ expression, thereby leading to the tumorigenesis of OS. 
signaling, indicating that miR-146a might promote OS tumorigenicity via targeting $\mathrm{ZNRF} 3 / \mathrm{GSK}-3 \beta / \beta$-catenin signaling (Figure 8).

Taken together, our study demonstrates that miR146a is highly expressed in OS tissues and has the positive correlation with tumor size and high recurrence of OS patients. Restoration of miR-146a expression promotes OS cell growth via targeting ZNRF3/GSK-3 $\beta / \beta$-catenin pathway, and represents a promising biomarker for OS patients.

\section{MATERIALS AND METHODS}

\section{Materials}

OS cell lines (U-2OS, Saos-2, MG-63, SW-1353, HOS, 143B) used in this study were from Laboratory of Orthopaedic Surgery of Renji Hospital. The materials and reagents used in this study were summarized in Supplementary Table 4.

\section{Cell culture and transfection}

Cell resuscitation and frozen are manipulated according to the standard process. OS cells were cultured with DMEM medium added with $10 \%$ FBS and 1\% penicillin $(100 \mathrm{U} / \mathrm{ml})$ and streptomycin $(100 \mu \mathrm{g} / \mathrm{ml})$ in a humidified atmosphere containing $5 \% \mathrm{CO} 2$ at $37^{\circ} \mathrm{C}$. Lentivirus-mediated miR-146a or miR-146 shRNA vector construction and cell transfection were referred as previously described [39]. The transfection efficiency of miR-146 or miR-146a shRNA were determined by qPCR.

\section{Quantitative real-time PCR (qRT-PCR)}

Total RNA exaction, reverse transcription and cDNA amplification were manipulated depending on the protocols provided by the manufacturers, of which miR-146a exaction was based on the protocols provided by miRNeasy mini kit. The primers of miR-146a, U6, ZNRF3 and GAPDH were listed in Supplementary Table 5. Data were analysed using the comparative $\mathrm{Ct}$ method $\left(2^{-\Delta \Delta C t}\right)$. Three separate experiments were performed for each clone.

\section{Western blot analysis}

OS cells and xenograft tumor tissue cells were harvested and extracted using lysis buffer (Tris- $\mathrm{HCl}$, SDS, Mercaptoethanol, Glycerol)., and the experimental protocols for western blot analysis was performed as previously reported [40].

\section{Cell viability assay}

OS cells transfected with miR-146a, miR-146a shRNA and ZNRF3 were incubated in 96-well-plates at a density of $2 \times 103$ cells. Cells were pretreated with $10 \mu \mathrm{l}$ of MTT dye each day. After incubation for $4 \mathrm{~h}, 100 \mu \mathrm{l}$ of DMSO were added into the cells for $15 \mathrm{~min}$. The color reaction was measured at $570 \mathrm{~nm}$ using an Enzyme Immunoassay Analyzer (Bio-Rad, Hercules, CA).

\section{Colony formation assay}

OS cells transfected with miR-146a, miR-146a shRNA and ZNRF3 were trypsinized and reseeded into 6-well plate. The number of cell colonies in control and treatment groups were counted after a week and were stained with crystal violet.

\section{Dual-luciferase reporter assay}

OS cells were cultured in 24-well plates. Gene report vector containing wild type 3 '-UTR or mutated 3'-UTR of ZNRF3 target gene was co-transfected with negative control or miR-146a into the OS cells. The detained manufacturer's instructions were referred to previously published [41].

\section{Animal experiments}

Six-week-old female immune-deficient nude mice were fed in Animal Institute of Chinese Academy of Sciences based on the regulations and internal biosafety and bioethics guidelines of Renji Hospital, Shanghai Jiao Tong University. Mice were injected subcutaneously with $1 \times 10^{6}$ U-2OS cells stably transfected with sh-miR-146a or control cells. The length and width of the Xenograft tumors was measured with a caliper every other day.

\section{Statistical analysis}

SPSS 21.0 was applied for the statistical analysis of the values, which were recorded as the mean \pm SEM. Paired or independent $t$ test was used to analyse the differential change in each group. Statistical significance was set at $P<0.05$.

\section{ACKNOWLEDGMENTS}

This research was funded by Shanghai Pujiang Program (NO.15PJD026), Medical-Engineering Joint Fund of Shanghai Jiao Tong University (NO. YG2014MS51), Shanghai Fund for Young Physician Development (NO.20141051), Incubating Program for Clinical Research and Innovation Of Renji Hospital (NO. PYXJS16-006)

\section{CONFLICTS OF INTEREST}

The authors declare no conflicts of interest. 


\section{REFERENCES}

1. Ene R, Sinescu RD, Ene P, Popescu D, Cîrstoiu MM, Cîrstoiu FC. Proximal tibial osteosarcoma in young patients: early diagnosis, modular reconstruction. Rom J Morphol Embryol. 2015; 56:413-7.

2. Liebner DA. The indications and efficacy of conventional chemotherapy in primary and recurrent sarcoma. J Surg Oncol. 2015; 111:622-31.

3. Durfee RA, Mohammed M, Luu HH. Review of osteosarcoma and current management. Rheumatol Ther. 2016; 3:221-43.

4. Ji W, Sun B, Su C. Targeting microRNAs in cancer gene therapy. Genes (Basel). 2017; 8:pii:E21.

5. Xiao B, Zhu ED, Li N, Lu DS, Li W, Li BS, Zhao YL, Mao XH, Guo G, Yu PW, Zou QM. Increased miR-146a in gastric cancer directly targets SMAD4 and is involved in modulating cell proliferation and apoptosis. Oncol Rep. 2012; 27:559-66.

6. Garcia AI, Buisson M, Bertrand P, Rimokh R, Rouleau E, Lopez BS, Lidereau R, Mikaélian I, Mazoyer S. Downregulation of BRCA1 expression by miR-146a and miR$146 \mathrm{~b}-5 \mathrm{p}$ in triple negative sporadic breast cancers. EMBO Mol Med. 2011; 3:279-90.

7. Burger ML, Xue L, Sun Y, Kang C, Winoto A. Premalignant PTEN-deficient thymocytes activate microRNAs miR$146 \mathrm{a}$ and miR-146b as a cellular defense against malignant transformation. Blood. 2014; 123:4089-100.

8. Czajka AA, Wójcicka A, Kubiak A, Kotlarek M, BakułaZalewska E, Koperski Ł, Wiechno W, Jażdżewski K. Family of microRNA-146 regulates RAR $\beta$ in papillary thyroid carcinoma. PLoS One. 2016; 11:e0151968.

9. Yamashita J, Iwakiri T, Fukushima S, Jinnin M, Miyashita A, Hamasaki T, Makino T, Aoi J, Masuguchi S, Inoue Y, Ihn $\mathrm{H}$. The rs2910164 $\mathrm{G}>\mathrm{C}$ polymorphism in microRNA-146a is associated with the incidence of malignant melanoma. Melanoma Res. 2013; 23:13-20.

10. Hsieh JY, Huang TS, Cheng SM, Lin WS, Tsai TN, Lee OK, Wang HW. miR-146a-5p circuitry uncouples cell proliferation and migration, but not differentiation, in human mesenchymal stem cells. Nucleic Acids Res. 2013; 41:9753-63.

11. Elsarraj HS, Stecklein SR, Valdez K, Behbod F. Emerging functions of microRNA-146a/b in development and breast cancer: microRNA-146a/b in development and breast cancer. J Mammary Gland Biol Neoplasia. 2012; 17:79-87.

12. Forloni M, Dogra SK, Dong Y, Conte D Jr, Ou J, Zhu LJ, Deng A, Mahalingam M, Green MR, Wajapeyee N. miR146a promotes the initiation and progression of melanoma by activating Notch signaling. Elife. 2014; 3:e01460.

13. Xu L, Zhong H, Wan H, Chen FY, Zhong J, Xiao F, Liu J, Shen L. miR-146a expression level as a novel putative prognostic marker for acute promyelocytic leukemia. Dis Markers. 2014; 2014:150604.
14. Sandhu R, Rein J, D’Arcy M, Herschkowitz JI, Hoadley KA, Troester MA. Overexpression of miR-146a in basallike breast cancer cells confers enhanced tumorigenic potential in association with altered p53 status. Carcinogenesis. 2014; 35:2567-75.

15. Paik JH, Jang JY, Jeon YK, Kim WY, Kim TM, Heo DS, Kim CW. MicroRNA-146a downregulates NFkB activity via targeting TRAF6 and functions as a tumor suppressor having strong prognostic implications in NK/T cell lymphoma. Clin Cancer Res. 2011; 17:4761-71.

16. Hou Z, Xie L, Yu L, Qian X, Liu B. MicroRNA-146a is down-regulated in gastric cancer and regulates cell proliferation and apoptosis. Med Oncol. 2012; 29:886-92.

17. Xu B, Wang N, Wang X, Tong N, Shao N, Tao J, Li P, Niu X, Feng N, Zhang L, Hua L, Wang Z, Chen M. MiR-146a suppresses tumor growth and progression by targeting EGFR pathway and in a p-ERK-dependent manner in castration-resistant prostate cancer. Prostate. 2012; $72: 1171-8$.

18. Lerner C, Wemmert S, Bochen F, Kulas P, Linxweiler M, Hasenfus A, Heinzelmann J, Leidinger P, Backes C, Meese E, Urbschat S, Schick B. Characterization of miR-146a and miR-155 in blood, tissue and cell lines of head and neck squamous cell carcinoma patients and their impact on cell proliferation and migration. J Cancer Res Clin Oncol. 2016; 142:757-66.

19. Zu Y, Yang Y, Zhu J, Bo X, Hou S, Zhang B, Qiu J, Zheng J. MiR-146a suppresses hepatocellular carcinoma by downregulating TRAF6. Am J Cancer Res. 2016; 6:2502-13.

20. Peta E, Cappellesso R, Masi G, Sinigaglia A, Trevisan M, Grassi A, Di Camillo B, Vassarotto E, Fassina A, Palù $\mathrm{G}$, Barzon L. Down-regulation of microRNA-146a is associated with high-risk human papillomavirus infection and epidermal growth factor receptor overexpression in penile squamous cell carcinoma. Hum Pathol. 2017; 61:33-40.

21. Li YL, Wang J, Zhang CY, Shen YQ, Wang HM, Ding L, Gu YC, Lou JT, Zhao XT, Ma ZL, Jin YX. MiR-146a-5p inhibits cell proliferation and cell cycle progression in NSCLC cell lines by targeting CCND1 and CCND2. Oncotarget. 2016; 7:59287-98. https://doi.org/10.18632/ oncotarget.11040.

22. Mei J, Bachoo R, Zhang CL. MicroRNA-146a inhibits glioma development by targeting Notch1. Mol Cell Biol. 2011; 31:3584-92.

23. Zhang X, Li D, Li M, Ye M, Ding L, Cai H, Fu D, Lv Z. MicroRNA-146a targets PRKCE to modulate papillary thyroid tumor development. Int J Cancer. 2014; 134:257-67.

24. Sun Q, Zhao X, Liu X, Wang Y, Huang J, Jiang B, Chen Q, Yu J. miR-146a functions as a tumor suppressor in prostate cancer by targeting Rac1. Prostate. 2014; 74:1613-21. 
25. Kogo R, Mimori K, Tanaka F, Komune S, Mori M. Clinical significance of miR-146a in gastric cancer cases. Clin Cancer Res. 2011; 17:4277-84.

26. Li H, Xie S, Liu M, Chen Z, Liu X, Wang L, Li D, Zhou Y. The clinical significance of downregulation of mir-124-3p, mir-146a-5p, mir-155-5p and mir-335-5p in gastric cancer tumorigenesis. Int J Oncol. 2014; 45:197-208.

27. Wu C, Cao Y, He Z, He J, Hu C, Duan H, Jiang J. Serum levels of miR-19b and miR-146a as prognostic biomarkers for non-small cell lung cancer. Tohoku J Exp Med. 2014; 232:85-95.

28. Xu X, Yang X, Ru G, Wu Y, Zhang S, Xing C, Wu Y, Cao J. miR-146a gene polymorphism rs2910164 and the risk of digestive tumors: A meta-analysis of 21 case-control studies. Oncol Rep. 2014; 31:472-9.

29. Iguchi T, Nambara S, Masuda T, Komatsu H, Ueda M, Kidogami S, Ogawa Y, Hu Q, Sato K, Saito T, Hirata H, Sakimura S, Uchi R, et al. miR-146a polymorphism (rs2910164) predicts colorectal cancer patients' susceptibility to liver metastasis. PLoS One. 2016; 11:e0165912.

30. Li Y, VandenBoom TG, Wang Z, Kong D, Ali S, Philip PA, Sarkar FH. Up-regulation of miR-146a contributes to the inhibition of invasion of pancreatic cancer cells. Cancer Res. 2010; 70:5703.

31. Bhaumik D, Scott GK, Schokrpur S, Patil CK, Campisi J, Benz CC. Expression of microRNA-146 suppresses NF-kappaB activity with reduction of metastatic potential in breast cancer cells. Oncogene. 2008; 27:5643-7.

32. Wang C, Zhang W, Zhang L, Chen X, Liu F, Zhang J, Guan S, Sun Y, Chen P, Wang D, Un Nesa E, Cheng Y, Yousef GM. miR-146a-5p mediates epithelial-mesenchymal transition of oesophageal squamous cell carcinoma via targeting Notch2. Br J Cancer. 2016; 115:1548-54.

33. Zhou Y, Lan J, Wang W, Shi Q, Lan Y, Cheng Z, Guan H. ZNRF3 acts as a tumour suppressor by the Wnt signalling pathway in human gastric adenocarcinoma. J Mol Histol. 2013; 44:555-63.
34. Qiu W, Yang Z, Fan Y, Zheng Q. ZNRF3 is downregulated in papillary thyroid carcinoma and suppresses the proliferation and invasion of papillary thyroid cancer cells. Tumour Biol. 2016; 37:12665-72.

35. Bond CE, McKeone DM, Kalimutho M, Bettington ML, Pearson SA, Dumenil TD, Wockner LF, Burge M, Leggett BA, Whitehall VL. RNF43 and ZNRF3 are commonly altered in serrated pathway colorectal tumorigenesis. Oncotarget. 2016; 7:70589-600. https://doi.org/10.18632/ oncotarget. 12130 .

36. Hao HX, Xie Y, Zhang Y, Charlat O, Oster E, Avello M, Lei H, Mickanin C, Liu D, Ruffner H, Mao X, Ma Q, Zamponi $\mathrm{R}$, et al. ZNRF3 promotes Wnt receptor turnover in an R-spondin-sensitive manner. Nature. 2012; 485:195-200.

37. Shi J, Jiang X, Yu Z, He G, Ning H, Wu Z, Cai Y, Yu $\mathrm{H}$, Chen A. ZNRF3 contributes to the growth of lung carcinoma via inhibiting $\mathrm{Wnt} / \beta$-catenin pathway and is regulated by miR-93. Tumour Biol. 2016; 37:3051-7.

38. Wang Z, Wang Y, Ren H, Jin Y, Guo Y. ZNRF3 inhibits the invasion and tumorigenesis in nasopharyngeal carcinoma cells by inactivating the Wnt/ $\beta$-catenin pathway. Oncol Res. 2017; 25:571-7.

39. Comer BS, Camoretti-Mercado B, Kogut PC, Halayko AJ, Solway J, Gerthoffer WT. MicroRNA-146a and microRNA$146 \mathrm{~b}$ expression and anti-inflammatory function in human airway smooth muscle. Am J Physiol Lung Cell Mol Physiol. 2014; 307:L727-34.

40. Zhang J, Wang G, Chu SJ, Zhu JS, Zhang R, Lu WW, Xia LQ, Lu YM, Da W, Sun Q. Loss of large tumor suppressor 1 promotes growth and metastasis of gastric cancer cells through upregulation of the YAP signaling. Oncotarget. 2016; 7:16180-93. https://doi.org/10.18632/ oncotarget. 7568 .

41. Li Z, Meng Q, Pan A, Wu X, Cui J, Wang Y, Li L. MicroRNA-455-3p promotes invasion and migration in triple negative breast cancer by targeting tumor suppressor EI24. Oncotarget. 2017; 8:19455-66. https://doi. org/10.18632/oncotarget.14307. 